\title{
Fatal Adult Necrotizing Enterocolitis Associated with Fecal Impaction Case Presentation and Short Review
}

Mohamed Saleh*, Philippe Pauquet and Didier Thévenin

Intensive Care Unit, Centre Hospitalier d'Arras, Arras, France

\begin{abstract}
lleus is a frequent side effect of drugs having anticholinergic properties. Both old and new atypical neuroleptics have anticholinergic properties. Necrotizing enterocolitis associated with long-term neuroleptic treatment is a rare but potentially fatal condition sometimes presenting with initial "benign" clinical and biological features. We present the case of a 29 years old patient presenting with acute abdomen while being treated for major depression by a combination of neuroleptics and two other drugs having anticholinergic properties. The diagnosis of necrotizing enterocolitis related to fecal impaction was confirmed by abdominal Computerized Tomography (CT) Surgical management was difficult because of absence of macroscopic features of bowel ischemia or necrosis despite the presence of severe refractory septic shock. The patient died 4 days after hospital admission.
\end{abstract}

Keywords: Necrotizing enterocolitis in adults; Neuroleptics; Constipation

\section{Background}

Necrotizing enterocolitis in adults is rare and has been associated with post operative ileus more than forty years ago [1,2]. More recently, drugs having anticholinergic properties -especially neuroleptics-have been associated with necrotizing enterocolitis. This potentially fatal [3] side effect has been associated with phenothiazines as well as new atypical neuroleptics [4-6]. By reviewing medical and surgical literature we could not find consensus about definition, diagnosis and management of neuroleptics-associated necrotizing enterocolitis. This might be due to disease rarity and recognition only via isolated case reports or series. However, this rare but potentially fatal condition might be challenging for medical and surgical teams. Here we report a fatal case of documented neuroleptics associated necrotizing enterocolitis without obvious macroscopi evidence of intestinal necrosis or ischaemia despite two abdominal surgical explorations.

\section{Case Presentation}

A 29 years old female patient presented with acute abdominal pain and vomiting in our hospital emergency room. She complained of symptomatology, which included alternating diarrhea and constipation in the week before admission in our hospital. Her surgical past history was non-contributory. She had a long-term medical history of major depression and several suicide attempts. She was admitted in residential care facility seven days ago for treatment adjustment. Her treatment consisted of a combination of one tricyclic

Antidepressant, two phenothiazine-based neuroleptics and prophylactic anti-tremors treatment with one anticholinergic agent.

No stool softener or recent antibiotic treatment figured in her treatment. In emergency room the patient was obnubilated, Glasgow coma scale score was $12 / 15$, pulse rate was 145 beats per minute, blood pressure was $90 / 45 \mathrm{mmhg}$, central body temperature was 37 , $1^{\circ} \mathrm{C}$ and oxygen saturation was $97 \%$ with no oxygen supply needed. Body weight and height were $52 \mathrm{~kg}$ and $145 \mathrm{~cm}$, respectively. Her abdomen was markedly is tended and painful on superficial and deep palpation. Shortly after emergency room admission, the patient collapsed and became comatose. She needed fluid resuscitation and nor epinephrine infusion. The diagnosis of abdominal related septic shock was suspected. The patient was sedated, incubated and mechanically ventilated.

\section{Initial Investigations and Management}

Arterial blood gases analysis results were as follow: $\mathrm{pH} 7,18, \mathrm{PO}_{2}$ $96 \mathrm{mmhg}, \mathrm{PCO}_{2} 39 \mathrm{mmhg}$, base excess $-13 \mathrm{mmol} / \mathrm{l}, \mathrm{HCO}_{3}-14 \mathrm{mmol} / \mathrm{l}$. Initial blood lactate was $3 \mathrm{mmol} / \mathrm{l}$ (Our laboratory reference value is < $2 \mathrm{mmol} / \mathrm{l})$. Kidney and liver function tests were within normal range. Serum lipase and amylase were also within normal range. Serum procalcitonin was $1,9 \mathrm{ng} / \mathrm{ml}$ (Normal range $<0,5 \mathrm{ng} / \mathrm{ml}$ ). Complete blood count showed hemoconcentration ( $\mathrm{Hb} 16 \mathrm{~g} / \mathrm{dl}$ ) and mild hyperleukocytosis (white blood cells count $18000 / \mathrm{mm}^{3}$ ).

Blood coagulation tests were within normal range.Pregnancy test was negative. Chest X-rays, urine analysis and lumbar puncture showed no signs of extra-abdominal sepsis. Contrast-enhanced abdominal CT (Figures 1 and 2) showed important small and large intestine distension associated with multiple zones of fecal impaction. No signs of perforation or peritonitis were present. Our local surgical team recommended conservative medical treatment which consisted of hemodynamic support, large spectrum antibiotic treatment and nasogastric tube suction. The patient was then admitted in ICU for management.

\section{First day investigations, follow-up and management}

Serial blood cultures were negative. Stool culture for salmonella, shigella, yersinia, campylobacter and c.perfringens was negative. C.difficile A and B toxins were absent. Serum procalcitonin and lactate values rose to $55 \mathrm{ng} / \mathrm{ml}$ and $6 \mathrm{mmol} / \mathrm{l}$ respectively. On the contrary, complete blood count showed 8000 leukocytes $/ \mathrm{mm}^{3}$ and kidney and liver function tests as well as blood coagulation tests remained unchanged. The patient had persistent severe circulatory failure in relation to severe vasoplegic shock documented by echocardiography. Large spectrum antibiotic treatment by a piperacillin-tazobactam combination in association with amikacin was given. Optimal

${ }^{*}$ Corresponding author: Mohamed Saleh, Intensive Care Unit, Centre Hospitalier d'Arras, France, E-mail: bpco20032002@yahoo.fr

Received December 12, 2013; Accepted December 28, 2013; Published January 07, 2014

Citation: Saleh M, Pauquet P, Thévenin D (2014) Fatal Adult Necrotizing Enterocolitis Associated with Fecal Impaction Case Presentation and Short Review. Surgery Curr Res 4: 156. doi:10.4172/2161-1076.1000156

Copyright: $\odot 2014$ Saleh M, et al. This is an open-access article distributed under the terms of the Creative Commons Attribution License, which permits unrestricted use, distribution, and reproduction in any medium, provided the original author and source are credited. 


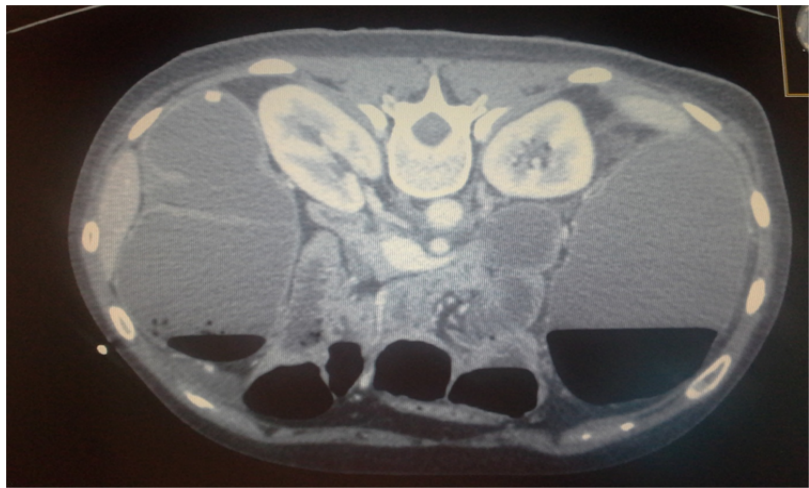

Figure 1: Contrast-enhanced abdominal CT

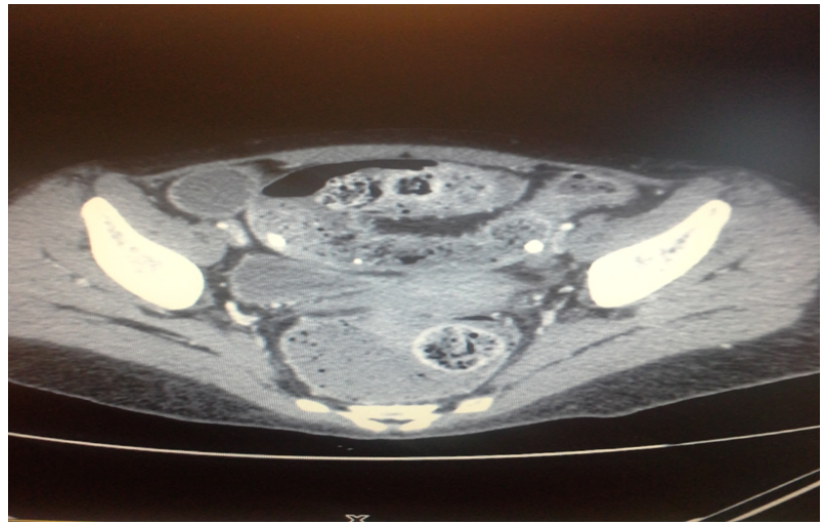

Figure 2: Contrast-enhanced abdominal CT

norepinephrine needed dose rose rapidly to reach $15 \mathrm{mg} / \mathrm{h}$. Our ICU team proposed urgent surgical exploration of the abdomen. Laparatomy showed no areas of macroscopic intestinal ischaemia or necrosis despite severe circulatory failure. Exploration of internal genital apparatus yielded normal findings. A small amount of clear peritoneal fluid was found. No intestinal resection was done. Peritoneal fluid culture remained negative.

\section{Next two days investigations, follow-up and management}

Blood tests showed persistent metabolic lactic acidosis, diffuse intravascular coagulation and leukopenia (white blood cells count $1200 / \mathrm{mm}^{3}$ ). Bacteroides uniformis was isolated in one blood culture made on the third day of admission. On the contrary, liver and renal function tests remained unchanged despite a worsening haemodynamic failure. A second contrast-enhanced abdominal CT showed diffuse thickening and infiltration of small and large intestine wall in addition to pneumatosis intestinalis in the ileum and the ascending colon (Figure 3) these findings were highly suggestive of necrotizing enterocolitis. A second look surgery was attempted on the third day. Macroscopic examination of the whole bowel was again noncontributory. The patient presented refractory shock and died on the fourth day of admission. Autopsy couldn't be done.

\section{Discussion}

The relation between fecal impaction, mental illness and necrotizing enterocolitis was underlined by Bos et al. [7] in a case series of three patients treated with neuroleptics. All patients were victims of food borne outbreak of enterotoxigenic C.perfringens typeA infection.
Two out of their three patients died. Few weeks later Sobel et al. [8] presented a second case series of four previously healthy adults who were victims of C.perfringens type A necrotizing enterocolitis related to food borne illness. Three patients out of four died. Despite differences between patients populations theses two case series reported a high mortality rate and pointed out the possible role of C.perfringens type A as causative agent of adult necrotizing enterocolitis with or without fecal impaction. Y reviewing other recent neuroleptics-associated necrotizing enterocolitis, the presence of enterotoxigenic C.perfringens type A seemed not to be mandatory for disease occurrence $[5,6]$. One possible mechanism of disease occurrence in such cases could be prolonged gastrointestinal hypo motility and constipation due to anticholinergic side-effect. This leads to ischemia by increasing intraluminal pressure and compression of mucosal vessels. As a sequel, bacterial translocation, inflammation and ischemia of the segment of bowel proximal to the obstruction. In our patient no specific bacterial agent could be identified. The occurrence of Bacteroides uniformis septicaemia late in the disease course might reflect bacterial translocation due to alteration of intestinal barrier and high intestinal anaerobic bacterial load. The absence of food borne outbreak illness in the patient's residential care facility and the absence of C.perfringens on stool culture argue against implication of C.perfringens type A in the disease pathogenesis.

By opposition to cases reported by Bos et al., [7] the macroscopic examination of our patient's small and large bowel on laparotomy was "normal" despite the presence of signs of small and large bowel necrosis on abdominal CT and severe abdominal related septic shock. A similar finding was reported by Rathat et al., who treated their patient with hyperbaric oxygen therapy [1]. This highlights the importance of mucosal examination to guide surgical resection of the necrotic segment. Mucosal examination in cases of bowelischaemia associated with acute vascular occlusion is usually done before surgery to guide necrotic intestinal segment resection. This would not be feasible for obvious reasons in cases associated with fecal impact. In the presence of organ failure, when macroscopic examination is "normal" and necrotizing enterocolitis related to fecal impaction is documented on abdominal CT examination several questions need answers:

- Could CT-guided intestinal resection be a reasonable option?

- $\quad$ Could repeated macroscopic examinations by one or more

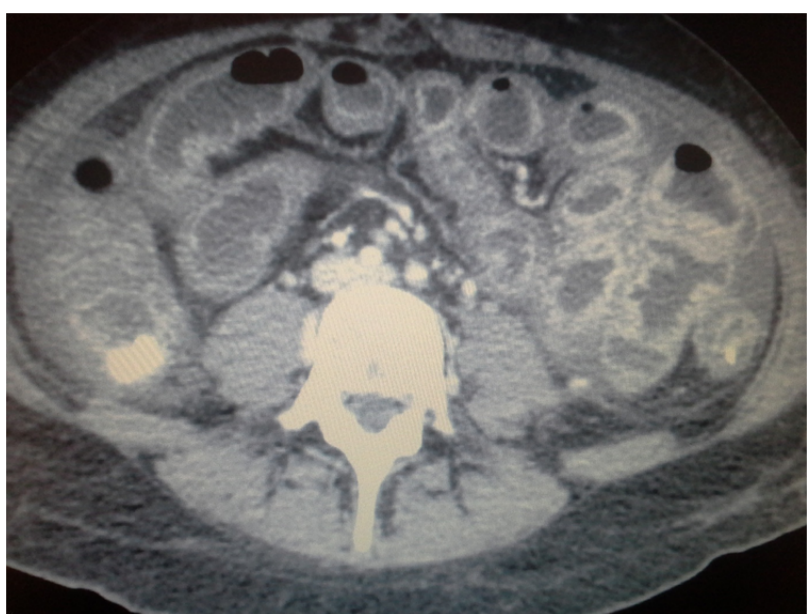

Figure 3: A second contrast-enhanced abdominal CT showed diffuse thickening and infiltration of small and large intestine wall in addition to Pneumatosis intestinalis in the ileum and the ascending colon 
Citation: Saleh M, Pauquet P, Thévenin D (2014) Fatal Adult Necrotizing Enterocolitis Associated with Fecal Impaction Case Presentation and Short Review. Surgery Curr Res 4: 156. doi:10.4172/2161-1076.1000156

surgical

- Explorations are helpful to unmask necrotic segments?

- $\quad$ Finally, could initial CT-guided resection followed by a second or third look surgery be life-saving?

Until answering these questions, clinician should be aware of this potentially fatal condition presenting sometimes with initial benign clinical picture. In the absence of definite medical and surgical management guidelines, surgical management of necrotizing enterocolitis associated with fecal impaction depends on operator experience and skills especially in borderline cases.

Finally, the bowel health of patients receiving medications that may cause constipation should closely monitored, and stool softeners should be prescribed as needed. The term neuroleptics-associated necrotizing enterocolitis might be misleading. A more accurate descriptive name of the the disease would be "Constipation-associated necrotizing enterocolitis".

\section{References}

1. Rathat C, Gatt MT, Pourriat JL, Hoang P, Cupa M (1979) [Post-operative necrotisingenterocolitis. One case cured by hyperbaric oxygenotherapy (author's transl)]. Anesth Analg (Paris) 36: 71-74
2. Magnin G, Partensky C, Lévi J, Miellet C, Dargent D (1979) [Necrotisingenterocolitis and pregnancy. One case (author's transl)]. J Gynecol Obstet Biol Reprod (Paris) 8: 329-331.

3. Ellis P, McLean RM, Harrison-Woolrych H (2007) Clozapine: fatal 'constipation' more common than fatal agranulocytosis. New Zealand Medicines and Medical Devices Safety Authority. Prescriber Update 28: 7.

4. Ausseur A, Leroy C, Bazin B, Sarraz-Bournet B, Oureib J, et al. (1995) [Acute necrotizing enterocolitis during a prolonged treatment with neuroleptics] Presse Med 24: 577-579.

5. Khaldi S, Gourevitch R, Matmar M, Llory A, Olié JP, et al. (2005) Necrotizing enterocolitis after antipsychotic treatment involving clozapine and review of severe digestive complications -- a case report. Pharmacopsychiatry 38: 220 221.

6. Ikai S, Suzuki T, Uchida H, Mimura M, Fujii Y (2013) Reintroduction of clozapine after perforation of the large intestine--a case report and review of the literature. Ann Pharmacother 47: e31.

7. Bos J, Smithee L, McClane B, Distefano RF, Uzal F, et al. (2005) Fata necrotizing colitis following a foodborne outbreak of enterotoxigenic Clostridium perfringens type A infection. Clin Infect Dis 40: e78-83.

8. Sobel J, Mixter CG, Kolhe P, Gupta A, Guarner J, et al. (2005) Necrotizing enterocolitis associated with clostridium perfringens type $A$ in previously healthy north american adults. J Am Coll Surg 201: 48-56. 